\title{
Tangence
}

\section{Vagabondages au nom de Babel : l'onomastique et les figures de l'exil}

\section{Jean-Pierre Vidal}

Numéro 43, mars 1994

Babel et après : Paul Auster

URI : https://id.erudit.org/iderudit/025804ar

DOI : https://doi.org/10.7202/025804ar

Aller au sommaire du numéro

Éditeur(s)

Tangence

ISSN

0226-9554 (imprimé)

1710-0305 (numérique)

Découvrir la revue

Citer cet article

Vidal, J.-P. (1994). Vagabondages au nom de Babel : l'onomastique et les figures de l'exil. Tangence, (43), 98-115. https://doi.org/10.7202/025804ar d'utilisation que vous pouvez consulter en ligne.

https://apropos.erudit.org/fr/usagers/politique-dutilisation/ 


\section{Vagabondages au nom de Babel: l'onomastique et les figures de l'exil \\ Jean-Pierre Vidal}

Let me call myself, for the present, William Wilson. The fair page now lying before me need not be sullied by my real appellation.

\section{Edgar Allan Poe, William Wilson}

Call me Ishmael. Some years ago never mind how long precisely - having little or no money in my purse, and nothing particular to interest me on shore, I thought I would sail about a little and see the watery part of the world.

\section{Herman Melville, Moby Dick}

Call me Jonah. My parents did, or nearly did. They called me John.

Jonah - John - if I had been a Sam, I would have been a Jonah still - not because I have been unlucky for others but because somebody or something has compelled me to be certain places at certain times, without fail.

\section{Kurt Vonnegut, Jr., Cat's Cradle}

Comment s'appelaient-ils? Que vous importe? D'où venaient-ils? Du lieu le plus prochain. Où allaient-ils? Est-ce que l'on sait où l'on va?

Denis Diderot, Jacques le fataliste 
Qu'on me pardonne cette pléthore d'exergues. Elle offre le double intérêt d'illustrer on ne peut plus éloquemment que la question du nom c'est la question du lieu et que cette équation n'a peut-être nulle part autant qu'en Amérique servi de paraphe ou de balise à l'espace littéraire. En effet, que le pseudonyme agisse comme protecteur du lieu d'écriture (Poe), fasse signe intertextuel du proscrit de la Genèse, auquel un orgueilleux larguage d'amarres vient ici au contraire donner pleine possession de son destin (Melville), ou qu'à travers la citation ironique de Melville ${ }^{1}$ ce soit, à l'inverse, par l'entremise d'un nom tout aussi totémique et emprunté au même Good Book, la malheureuse appartenance du nom au lieu, la funeste coïncidence de l'inscription d'un corps dans un espace-temps, qui exemplairement se dise (Vonnegut), toujours l'imaginaire américain semble reprendre, au coeur même de ses plus folles envolées désirantes, ce geste de dénégation, de non-fondation, de non-inscription et notamment dans un paysage. Romulus, bien d'autres après lui, donne son nom à la ville qu'il fonde. Mais après que Vespucci y ait laissé la part la plus intime du sien, ce continent-ci fait au contraire tomber un à un tous les autres. Sans doute parce qu'il est encore l'image de la nouveauté absolue, de l'événement absolu, du monstre en un mot (voir Derrida à propos de Lautréamont). Et que, pour bon nombre, le monstre a nom exil. Le Melting pot fut d'ailleurs aussi une véritable centrifugeuse onomastique, un tordeur de noms propres qui rejetait dans un hors lieu oublieux non pas la totalité mais, ce qui peut-être est pire, des pans syllabiques entiers, trop peu anglo-saxons. Diminutif dès lors, le patronyme y devenait presque sobriquet, c'est-à-dire parole vive, non plus « désignateur rigide" pour reprendre l'expression de Kripke mais au contraire déjà phrase, parcours signifiant et donc programme narratif, à suivre ou à contredire, en tout cas impossible à ignorer.

\section{Le parcours du nom : palimpseste et palindrome}

Sans doute est-ce notamment cela qui s'illustre dans tel passage fameux de Moon Palace: "Later on, Uncle Victor told me

1 "Call me Redburn" ironisera une des lettres de Fanshawe (The Locked Room, troisième roman de The New York Trilogy). Cette double citation, cette citation condensée, n'est qu'une des nombreuses références à Melville qui parsèment l'œuvre d'Auster. 
100

that his father's name had originally been Fogelman, but someone in the immigration offices at Ellis Island had truncated it to Fog, with one $g$, and this had served as the family's American name until the second $g$ was added in 1907,2 (Penguin books, "Contemporary American Fiction", New York, 1990, p. 3. Toutes références ultérieures renverront à cette édition). La transmission avunculaire de l'histoire du nom comme figure de l'exil, encore pour la deuxième génération, témoignage métonymique (comme tous les témoignages), rupture et suture à la fois, aboutit à la fictionnalisation par lecture, vérité métaphorique au contraire, pour la troisième génération. Le nom propre aura été rendu à sa valeur originelle de nom commun, support et non aboutissement de toute fiction, comme il advient au contraire dans la glorification épique ou religieuse du nom. La liberté de l'imaginaire est ainsi rendue au neveu qui y voit confirmé le caractère volatile et métis (ou polyglotte) de son destin: "Fogel meant bird, my uncle informed me, and I liked the idea of having that creature embedded in who I was. I imagined that some valiant ancestor of mine had once actually been abble to fly, A bird flying through fog, I used to think, a giant bird flying across the ocean, not stopping until it reached America. ${ }^{3}$ (p. 3-4. C'est moi qui souligne).

Cette créature qui a "fait son lit" dans ce qu'il est lui-même, cet homme oiseau que dit l'allemand (le yiddish?), c'est à travers le redoublement-traduction, le calembour donc, de sa transposition anglaise, qu'il vole encore dans l'imaginaire de Marco. Étrange parcours du patronyme auquel l'amputation bisyllabique d'Ellis Island, la coupure de l'exil, qui pourtant gardait trace de l'oralité d'origine, le $v$ germanique transcrit "phonétiquement" en $f$, semble avoir donné des ailes: celles du redoublement du $g$ dû sans doute au grand-père, celles surtout du redoublement qu'opère le translinguistique comme perversion ludique du code,

2 "Plus tard, oncle Victor m'a raconté que le nom de son père était Fogelman, et que quelqu'un, à Ellis Island, dans les bureaux de l'immigration, l'avait réduit à Fog, avec un $\mathrm{g}$, ce qui avait tenu lieu de nom américain à la famille jusqu'à l'ajout du second g, en 1907. . (Moon Palace, traduction de Christine Le Bœuf, Arles, Actes Sud, 1990, p. 14)

3 "Fogel veut dire oiseau, m'expliquait mon oncle, et j'aimais l'idée qu'une telle créature fit partie de mes fondements. Je m'imaginais un valeureux ancêtre qui, un jour, avait été réellement capable de voler. Un oiseau volant dans le brouillard, me figurais-je, un oiseau géant qui traversait l'Océan sans se reposer avant d'avoir atteint l'Amérique." (ibid., p. 14) 
suture ou sublimation opérée par l'imaginaire, ce non-lieu qui jamais pourtant ne se laisse abstraire.

C'est ainsi dans le corps même de son nom que se déploie l'histoire mythique des ancêtres de Fogg, de même que la ville de Romulus, juste retour des choses, s'inventa vite une autre origine par la tutelle cachée dans l'anagramme palindrome (Roma-Amor) qui ainsi la vouait à Vénus.

Précisément, c'est de l'anagramme du nom qu'il est question dans ce passage de In the Country of Last Things, texte, on le sait, sensiblement contemporain de Moon Palace. Immigrant manifestement de la première génération dans ce pays imaginaire mais aussi proche de l'Amérique que le pays du Big Brotber d'Orwell l'était de l'Angleterre, Otto Frick, le chauffeur - factotum de Woburn House n'est pas, dans ses difficultés avec les mots, pour lui solides comme "des cailloux littéraux", sans évoquer paradoxalement Démosthène, dont l'éloquence était due, si l'on en croit la légende, à la façon dont il avait combattu un défaut de prononciation par l'habitude qu'il avait prise de répéter ses discours la bouche pleine de petits "cailloux", en fait des bonbons qu'on appelait ainsi. Otto Frick, en effet "had difficulty maneuvering (words) around his tongue, and he would sometimes stumble over them as though they were physical objects, literal stones cluttering his mouth "4 (Penguin Books, "Contemporary American Fiction", New York, 1988, p. 133). Dans une interprétation qui, sous nos climats littéraires, fait irrésistiblement penser à la thèse de Sartre dans L'idiot de la famille, la narratrice attribue à ces difficultés la sensibilité particulière qu'a Frick pour les "propriétés internes" des mots, "their sounds as divorced from their meanings, their symmetries and contradictions" (ibid.) ${ }^{5}$. Suit l'interprétation de Frick lui-même qui fait de nécessité vertu et, dans une dialectique bien connue des humiliés et offensés de tous bords et de toutes époques, retourne magiquement à son avantage le hasard a-signifiant de son nom, devenu par sa dynamique

4 "Il avait du mal à les mancuvrer autour de sa langue, et il lui arrivait de buter dessus comme s'il s'agissait d'objets solides, de pierres littérales obstruant sa bouche." (Le voyage d'Anna Blume, traduction de Patrick Ferragut, Arles, Actes Sud, coll. "Babel ", 1993, p. 181-182.)

5 "[...] il paraissait particulièrement sensible aux propriétés des mots en euxmêmes: de leur son en tant que distinct du sens, de leurs symétries et de leurs contradictions. " (ibid., p. 182) 
102

interne et le parcours littéral qu'elle ordonne, le signe d'une élection et le gage (rétrospectif, cela a son importance ironique) de sa longévité:

That's why I got to be such an old man. My name is Otto. It go back and forth the same. It don't end nowhere but begin again. I get to live twice that way, twice as long as no one else. You too, miss. You be named the same as me. A-n-n-a. Back and forth the same, just like Otto myself. That's why you got to be born again. (ibid. $)^{6}$

Attirée dans un piège en effet et menacée de mort, Anna Blume n'a eu d'autre recours que de se jeter par la fenêtre, laissée pour morte jusqu'à ce que les gens de Woburn House la ramassent dans la rue.

On sait qu'au Moyen-Âge des moines célébraient l'éternité de Dieu par la confection de palindromes étendus, équivalents littéraux de la mise en abyme héraldique, ou plus généralement visuelle, en ce sens que, comme elle, ils piègent l'infini dans un espace fini mais jouent sur le temps (de la lecture prise dans le cercle vicieux) quand elle repose d'abord sur l'espace et voit s'éteindre ses effets avec la limite qu'à la vue impose l'amenuisement puis la diminution de l'image qui la forme. C'est bien d'ailleurs dans ce sens d'un éternel retour ("it don't end nowhere but begin again") que Mr Frick interprète le palindrome de son prénom et de celui d'Anna.

Mais le temps humain n'est pas éternel, il n'est que double, dans sa durée (Frick) ou son recommencement (Anna). Et on aura noté que le palindrome d'Anna et d'Otto est prénominal et non patronymique et que, par ailleurs, il s'oppose au calembour translinguistique du fogel through fog en ce sens que si ce dernier peut être, à la rigueur, assimilé à un palindrome, c'est sur le mode "vertical" ou paradigmatique de la connotation, de la surimpression, bref du palimpseste plus que du palindrome, mais d'un palimpseste d'un genre particulier puisque c'est le seul changement de support (ici, paradoxalement, la langue) qui fait

6 "C'est pourquoi je suis devenu si vieux. Je m'appelle Otto. Ça va et ça revient pareil. Sans fin nulle part mais ça recommence. Comme ça, je peux vivre deux fois, deux fois plus longtemps que personne. Aussi vous, mademoiselle. Vous avez un nom comme moi, A-n-n-a. Ça va et ça revient pareil, comme Otto pour moi. C'est pourquoi vous êtes renée. " (ibid., p. 182) 
apparaître l'autre inscription, comme si à la feuille grattée d'un manuscrit unique s'était ici substituée la duplication des feuilles où s'inscrit l'écriture, elle-même demeurée plus ou moins inchangée. Ainsi l'écriture se présente-t-elle comme une moire de langues, la fluctuation babélienne d'un métissage linguistique ( $\mathrm{Ba}-$ bel", on le sait, vient de l'hébreu balal qui veut dire "confondre", "mêler"). Entre l'oiseau allemand et le brouillard anglais, les lettres communes, la lettre transcrite et les lettres perdues dessinent la solution de continuité que forme toute translation, toute translittération, en trois temps, qui sont comme ceux de la traduction: quelque chose reste, quelque chose se transforme, quelque chose se perd. Il serait aisé de montrer comment toute l'aventure de Fogg obéit à ces trois temps et comment, en particulier, de Zimmer (la pièce, la chambre en allemand) au restaurant Moon Palace, un système littéralement optique ${ }^{7}$ permet de relayer le parcours de l'ancêtre en l'inversant de façon parfaite: Fogg marchant à travers ses propres transformations (dépouillement puis richesse, ascèse et dépense), son ascendance retrouvée et l'Amérique qui permet tout cela ${ }^{8}$, ne s'arrêtant point avant d'avoir atteint l'Océan autre, c'est-à-dire, puisque paraît-il la terre est

7 Entre autres occurrences l'appartement vide qui permet d'un certain angle de voir l'enseigne du restaurant Moon Palace et la lune à la fin que Fogg, au bord du Pacifique, enfin arrivé sur terre en quelque sorte et sa vie recommençant comme celle de l'ancêtre Vogelman, peut voir "trouv(er) sa place dans les ténèbres". Elle aussi a-t-on envie d'ajouter, tant ce roman qui s'ouvre avec l'évocation de l'homme débarquant sur la lune, est tissé d'un impérieux réseau de trajectoires quasi orbitales. Avec Moon Palace, Auster aura réalisé l'inverse en quelque sorte, un livre symétrique, "pair" dirait Faustroll, des cuvres explicitement citées de Jules Verne et de Cyrano de Bergerac: une projection de la carte du ciel et du tracé rigoureux de ses mystérieuses trajectoires sur les mouvements browniens des corpuscules humains. Moon Palace pourrait fort bien se sous-titrer, en effet, l'arrivée sur terre de Marco Stanley Fogg, "man in the moon" du proverbe où l'anglais dit l'hurluberlu ou celui qui ignore tout de quelque chose plus que le pierrot ( $c f$. "I know nothing more of it than the man in the moon .)

8 Le rêve américain repose aussi en partie sur le double désir, contradictoire seulement en apparence, d'être quelqu'un, c'est-à-dire pas n'importe qui, et personne. Une sorte de Noli me tangere à la mode puritaine et démocratique. C'est ainsi, par exemple, que dans The Locked Room, Fanshawe fuira le regard de quiconque pourrait participer, en la cotoyant, à une identié qu'il veut intouchable parce qu'anonyme. Il abandonne donc statut social, femme et enfant. Et comme on ne saurait survivre longtemps dans l'anonymat, le pseudonyme est la tentation constante de tout américain bien né. Dans The Locked.Room, Fanshawe devient tout simplement le pseudonyme de son ami, le narrateur. 
104

ronde (la lune aussi), avant d'avoir pris à revers son ancêtre mythique, rien qu'en poursuivant le mouvement qu'il avait initié. En ce sens, on peut dire que Fogg est la plus petite mise en abyme du texte, puisque ce mouvement mythique qui sert d'armoirie au mouvement réel de l'immigration aboutit, par le mouvement de la lettre, à la fictionnalisation du hasard, l'origine perdue (vogelman) perçant de son mouvement même la nuée (fogg) que ce même mouvement, à un autre niveau, a produit. On ne saurait mieux dire que toute fiction tient à un glissement de lettres...ni que toute lettre est la trace tombée d'un parcours "réel". Entre les mots et les choses, il ne saurait être question d'antériorité, puisque les uns ne vont pas sans les autres, du moins pour l'espèce humaine qui se définit précisément par là. Circulation incessante, implication réciproque, l'univers d'Auster est incontestablement héraclitéen et l'on pourrait faire servir d'exergue à toute son œuvre ce mot de Rilke que Claude Simon a épinglé en épigraphe à Histoire: "Cela nous submerge. Nous l'organisons. Cela tombe en morceau. Nous l'organisons et tombons nous-mêmes en morceaux."

Remarquons par ailleurs que si le système onomastique d'Auster semble produire deux modalités concurrentes de fictionnalisation de la lettre, c'est probablement que le patronyme est généralement généalogique ou, mieux, phylogénique quand le prénom est plutôt (pseudo)individuel et ontogénique. Ce qui explique qu'on puisse lire le patronyme comme un mot qui dit le destin de la race quand on ne peut bien souvent qu'apprébender le prénom comme une chose dont les propriétés purement physiques et objectives fondent l'interprétation. Le patronyme passe par le code commun de la langue, redoublement de la généalogie (dans l'exemple de Fogg, le redoublement est aussi négation puisque la langue est double), tandis que le prénom est renvoyé au phénomène, à l'événement et à sa perception strictement individuelle, bien que tout ceci passe aussi, bien entendu, par la langue. Le patronyme est Histoire et en porte les marques (coupure, ajout, traduction), il est, pour reprendre la dichotomie saussurienne, langue morte que réanime la parole interprétative de Marco, intervention qui n'est pas sans évoquer l'action propre à la littérature à la fois sur la langue et sur sa propre histoire, ce qui a été écrit attendant toujours l'intervention de l'autre, de son déchiffrement, de son imaginaire, de sa lecture en un mot (parfois de son écriture, l'histoire littéraire elle-même se résumant à une 
suite de palimpsestes), pour advenir enfin tel qu'en lui-même toute re-production le change. Lire aussi c'est écrire dans sa propre généalogie. Le prénom, quant à lui, c'est presque, au contraire, le monde sans les hommes, un espace dont les lois sont internes et n'admettent qu'une leçon, celle d'un destin individuel ainsi mis à sa place par les lignes de force de l'univers: le doublement d'Otto n'est pas celui d'Anna. Le patronyme fait signe par le social, se déchiffre par la lecture commune, jouât-elle le translinguistique. Le prénom résonne comme un oracle et s'entend comme un destin parce qu'il est l'ordre secret du monde disposé en forme d'index du sujet. Il joue le sens comme vecteur contre la signification comme échange.

Ce sont là du moins les deux pôles du système onomastique d'Auster qui, en fait, est bien plus complexe que ces deux exemples extrêmes et symétriques du patronyme de Fogg et des prénoms d'Anna et d'Otto ne semblent le dire.

\section{Le renvoi intertextuel: prégnance de la pseudonymie.}

En ce qui a trait à Fogg, il faut aussitôt ajouter en effet que l'aboutissement du parcours du nom de l'ancêtre migrant n'est pas seulement l'agglomération hasardeuse de quatre lettres anonymes mais qu'aussitôt assemblées ces lettres transforment le nom propre du personnage en renvoi intertextuel et ainsi d'une certaine façon en pseudonyme: "Not long ago I arrived in Chicago, Uncle Victor took me to a showing of Around the world in 80 Days. The hero of that story was named Fogg, of course..." ${ }^{9}$ (ibid., p. 6). Ce "of course" est un poème, une pure décharge d'ironie postmoderne. Car il sanctionne l'évidente anamnèse de la lecture "lettrée" et ac-

9 "Peu de temps après mon arrivée à Chicago, oncle Victor m'a emmené voir le film à succès de la saison, Le tour du monde en quatre-vingts jours. Le nom du héros de cette histoire est Fogg, bien sûr..." (ibid., p. 17). C'est entendu, toute traduction est choix mais on se demande malgré tout où la traductrice a bien pu prendre ce "film à succès de la saison" qui donne une précision particulière à la temporalité de cette période de la vie de Fogg. Symétriquement, on questionnera le parti-pris étrange qui fait traduire, dans l'incipit, "I did not believe there would ever be a future" par "je n'avais aucune foi dans l'avenir". À part la référence escamotée au "no future " des punks, c'est une chose de ne pas croire en l'avenir et une autre chose de croire qu'il n'y aura pas d'avenir. Par d'imperceptibles libertés comme cellelà on modifie décidément un peu trop le système temporel du roman. 
106

corde à l'autre, si elle existe encore, la politesse pince-sans-rire d'un "comme chacun sait" adressé à qui ne sait pas. Dans les deux cas, c'est un "gotcha!" qui s'entend là. Et derrière le clin d'œil s'ouvre un abîme qui fait vaciller la lecture: dans une imperturbabilité digne de la 'Pataphysique et de ses zélotes collégiens, ce qui se dit alors, en effet, c'est le caractère incommensurable de la culture, l'indécidable de la citation une fois son trompe-l'oil mis en évidence, l'ubiquité obsessionnelle de l'intertexte une fois son processus explicitement représenté. Ce qui se dit, somme toute, c'est le hasard de la référence et les chausse-trappes de la re-connaissance, la coïncidence comme figure de discours et surtout métaphore de la lecture. Car qui m'assurera qu'ainsi parti l'auteur ne me révèle pas ce que je soupçonnais déjà pour mieux me cacher cette référence qui, elle, m'échappe et m'échappera toujours à cause du silence qu'il garde sur elle? Inversement, ainsi excitée, la projection de ma culture sur le texte, qui me garantira que c'est à bon droit que je vois le texte lui ménager une place. L'universalité du texte est ainsi minée par la menace de la private joke, celle de l'écriture et celle de la lecture.

Par cette mise à plat, cette surreprésentation de la référence comme tracé intime, de l'invention scripturale comme citation, du texte comme intertexte et de l'intertexte comme hasard sémiotique, Paul Auster offre à la fois une remarquable allégorie de la littérature (et du monde), un symptôme amusé de la "condition postmoderne" et peut-être, ce qui ne serait pas son moindre paradoxe, une illustration de ce qu'il faut bien appeler sa judaïté, une telle posture en effet n'étant pas sans évoquer l'extraordinaire littérature rabbinique que fonde, on le sait (of course), une conception foncièrement intertextuelle des textes sacrés comme supports de variations infinies qui toutes célèbrent un Dieu luimême présenté "écrivant "le monde ${ }^{10}$.

Mais revenons à Fogg, dont la rencontre, par cinéma interposé, du personnage homonyme de Jules Verne produit à la fois

10 Voir à ce sujet la superbe étude de Stéphane Zagdanski, L'impureté de Dieu, la lettre et le péché dans la pensée juive, Paris, Éditions du Félin, 1991, 234 p. On ne saurait d'ailleurs sous-estimer la part qu'a prise manifestement le judaisme comme courant de pensée, culture, lieu intellectuel, dans la conception contemporaine de l'écriture, ne serait-ce que par la signature de Jacques Derrida, entre autres (notamment Borgès, nourri, comme on sait, de la tradition rabbinique; Eco plus récemment, dans ses romans, etc.). 
un décollage de sa propre identité sociale par l'effet déréalisant de la pseudonymie, renforcée encore par l'oncle Victor qui dès lors lui donne le prénom du personnage de Verne, et une confirmation par surdétermination de son identité mythique : il sera décidément ce voyageur que le destin de l'ancêtre migrant, au bout de ses multiples transformations a inscrit dans son nom avec le filigrane de son origine tracé dans l'encre sympathique de l'étymologie et de la traduction (de la diachronie et de la synchronie, ou si l'on préfère, du temps et de l'espace) et que le dessein inventif d'un écrivain lui a tendu en guise de confirmation, dans l'imparable coup du sort d'une coincidence aussitôt devenue signe, index même. Ainsi histoire familiale et Histoire tout court (sous la forme de son avatar littéraire et du "of course" qui le ponctue), espace "réel" et espace fictif se trouvent conjoints, mais bien entendu à l'intérieur de la fiction du texte. C'est-à-dire que le parcours qui dans la fiction aboutit au nom de Fogg, accident heureux en forme de présage, est en fait, à rebours, dès le départ un choix d'écriture (disons, pour faire bref, celui de la variation intertextuelle) qui se donne, toujours à rebours, non pas le programme de prononcer, couper et traduire le nom prétendument ancestral, mais plutôt celui de faire pousser un prolongement non seulement littéral mais aussi "privé" (la fiction de l'histoire privée du personnage et de sa famille) à tel nom déjà attesté par l'histoire littéraire. Le nom hasard est en fait un nom mémoire et l'accident d'un parcours la fidélité du souvenir. Ou plutôt de l'anamnèse, mais d'une anamnèse active, comme si Paul Auster s'était plu à transcrire métaphoriquement dans l'ascendance de son héros la sienne propre: Österreich, Austria, l'Autriche, l'éponyme en quelque sorte. Signe on ne peut plus révélateur d'une ceuvre qui oscille toujours entre la confidence tacite et l'exubérance inventive, l'euphorie du récit et le deuil du silence, ce silence qui est à la fois musique et rature, comme Beckett l'a si admirablement montré en ouvrant les éternités tapies dans l'espace insondable de la lettre.

Quoi qu'il en soit, Fogg est donc dès le début du roman ce paradoxe: une identité qui ne se peut nommer que par une autre qu'elle n'est pas. Et le personnage de deux romans à la fois, l'inverse sur ce point du Don Quicbotte revu par Borgès et attribué par lui à l'improbable Pierre Ménard, deuxième auteur du même roman, dont on pourrait dire dès lors qu'il en admet, par le fait même, un troisième, Borgès lui-même. Si j'insiste sur ce point 
c'est qu'il me semble éclairer d'un jour particulièrement révélateur le geste de Paul Auster s'inscrivant nommément comme "personnage " et en tout cas pseudonyme dans City of Glass, avec la réciproque récente qu'est l'inscription du personnage Quinn dans la "vie" de l'«auteur" Paul Auster ( $c f$. The Red Notebook, titre qui bien entendu répercute le cahier rouge dans lequel le Quinn de City of Glass lui-même écrivait.)

Dans un cas, quelqu'un au téléphone demande à parler à Paul Auster mais c'est un certain Quinn qui répond, dans l'autre un certain Paul Auster répond à qui veut en fait parler à Quinn. Cherchez l'origine. Ou celui qui lance les dés. Car le premier Quinn, en fait, écrit des romans policiers, non pas sous le pseudonyme de Paul Benjamin qui cache en fait Paul Auster, mais sous celui de William Wilson (ce qui fait aussi de lui le personnage de deux récits à la fois) qui révèle le nom, en fait un pseudonyme, du personnage d'une histoire de double, une des plus belles, écrite par ce père putatif du roman policier, Edgar Allan Poe ${ }^{11} 11$. Nous sommes bien, qui ne le voit?, dans une des travées de la bibliothèque de Babel. Avançons davantage, là, approchons-nous de ce téléviseur allumé où un homme, regardant la caméra, dit: "Même Paul Auster n'est pas Paul Auster". Cet homme, c'est Paul Auster, à la fin de l'entrevue qu'il accordait à Stéphan Bureau.

Où en étais-je? Et même où est je? Évidemment tombé dans le gouffre de la lettre. Qui n'a pas de fond. Et pourrait pour nous

11 Nombreuses références à Poe, également, dans l'œuvre d'Auster, la plus importante restant sans doute, dans City of Glass, celle qui établit un parallèle entre la fin d'Artbur Gordon Pym et le tracé littéral formé par la déambulation urbaine de Stillman. Je reviendrai sur ce point. Qu'il suffise pour le moment de dire qu'elle prend place à côté de celles de Melville dans une relance intertextuelle de l'obsession du signe, y compris dans sa dimension morale, qui a marqué la grande littérature américaine de la première moitié du $\mathrm{XIX}^{\mathrm{e}}$ siècle et dont la culmination est peut-être The Confidence Man (1857) de Melville, fable (et farce) hallucinée du métissage des discours et du signifiant comme perpetuum mobile, leurre et simulation, à la limite sans objet. C'est le plus "postmoderne * des romans de Melville et l'un des plus méconnus du public francophone. Quoi qu'il en soit, la façon dont Auster réinscrit les grands textes de la littérature américaine mériterait, à elle seule, une étude (amorcée ici même par Daniel Canty) dont le point de départ pourrait être la jolie formule de Don De Lillo à son propos: "He blends modern surfaces with 19th-century interiors" (quatrième de couverture de Moon Palace). Surface, intérieur, la question du signe et de l'identité, encore une fois. 
Québécois emprunter les accents de la fin de Neige noire: "[...] Le temps me dévore, mais de sa bouche, je tire mes histoires, de sa sédimentation mystérieuse, je tire ma semence d'éternité. Eva et Linda approchent de ce thêâtre illuminé où la pièce qu'on représente est une parabole dans laquelle toutes les oeuvres humaines sont enchâssées. " ${ }^{12}$

C'est bien en effet de cet enchâssement fondamental, entendu également dans la célèbre phrase finale des Mots ("Un homme et qui les vaut tous et que vaut n'importe quin), qu'il est question ici, comme de ce qui est le point de départ de toute prise de parole, de toute écriture, de toute signature, de toute fiction même: le recours à l'Autre. L'Autre est le nom propre de cet enchâssement. Il est en effet à la fois cette adresse que je lui fais et cet appel qu'il fait de moi. Et nous sommes tous ses pseudonymes. Si la littérature a quelque sens, c'est bien d'être l'expérience privilégiée de cela qui n'a rien, mais alors là rien, à voir avec la petite robinetterie "émetteur-récepteur" dont on nous rebat les conduits, pour mieux rabattre nos conduites, peut-être.

\section{La voix de l'Autre : de la variation.}

Donc, nommer c'est donner le champ libre à l'Autre en le suscitant par cet acte fondateur de toute pratique artistique: la variation, qui est à la fois répétition, différence et hasard. Non seulement la variation est-elle au départ de la mimesis puisqu'il n'est pas de représentation sans épreuve de la distance, mais toute poiesis repose sur elle et rencontre à un moment de son dynamisme la limite extrême de la variation qu'est le hasard advenu, capturé, "charmé", en donnant à ce dernier terme son acception latine de "poétisé", ce qu'on pourrait appeler une poétique stochastique.

On trouve une étonnante représentation de cette infinité de la variation dans ce qu'il advient aux noms et prénoms de Fogg,

12 Hubert Aquin, Neige Noire, Montréal, La Presse, 1974, p.254. Je me dois de rappeler ici que des complexités inouies, sûrement prévues par l'auteur, empêchent ici le modeste citeur de respecter le texte et d'accomplir en rigueur son travail. Ce passage, en effet, est entre parenthèses dans le texte, enchâssement jusqu'où tu peux aller, et, ne prenant pas le contenu entier de cette parenthèse, je me devrais, pour bien citer, d'y inscrire ma parenthèse avec les trois petits points de rigueur dans la parenthèse initiale qu'ainsi j'ouvrirais dans la mienne. Enchâssement, quand tu nous tiens! 
110

dès lors qu'ils sont lancés dans l'espace public "réel", celui de l'école. Auparavant les "théories absurdes" de l'Oncle Victor auront trouvé une motivation secrète à l'arbitraire onomastique, et de telle façon qu'ainsi ancrés à leur référent les nom et prénoms du personnage affirmeront un double paradoxe: d'une part c'est, justement, cet "ancrage " de son nom qui le voue aux amarres larguées et en fait donc le contraire de l'Ishmael de Melville, d'autre part ce même ancrage identitaire est en fait la marque d'une pseudonymie intertextuelle qui à peine posée relance le nom dans la variation paronomastique. Voyons un peu en détail ce double paradoxe.

La mobilité que, selon l'oncle Victor, prédisent nom et prénom à leur porteur est le produit d'une immobilité référentielle, d'un épinglage; si Fogg est prédisposé au voyage c'est en effet à l'immobilité du rapport référentiel d'eux à lui qu'il le doit et l'on n'aura garde d'opposer cette permanence somme toute surprenante à la mobilité onomastique de son ascendance, non seulement l'ancêtre Vogelman mais aussi Barber et Effing. Celui-ci va même jusqu'à prendre pour nom un juron délité, dans un geste parfaitement symétrique de celui de l'homme oiseau: Vogelman se faisait couper son nom par "some half-literate American functionary" (p. 7), Effing lit à haute voix la coupure du sien, qui n'en est pas un, coupure où se marque la bienséance, la censure, sous la forme de ces trois points dont on atténue "fucking", les trois points de l'Autre qui ont aussi commencé d'américaniser Vogelman. Ainsi, le choix ironique, la revendication même, de la coupure sociale répond-il au décret indifférent de son hasard initial. Et, bien sûr, la transcription littérale du $f$ répond à sa traduction à partir de la phonie du $v$ germanique, comme si la langue ancestrale, toujours susceptible de traduction jusque dans son déni, aboutissait à la négation de toute langue qu'est la psalmodie littérale, le gramme refermé dès lors sur lui-même et n'admettant comme ouverture vers le monde, en lieu et place de tout signifié que l'autoréférentialité de son "nom" proféré.

Si Effing choisit librement un pseudonyme qui n'est même pas un nom, mais un participe, un juron, c'est-à-dire une marque purement phatique, Fogg se trouve par le hasard affublé d'un nom qui est un pseudonyme d'être déjà occupé, d'être une citation, tout entier au contraire perdu dans la dimension référentielle du langage. L'un marque sa présence par un titre qui revient en fin de compte à son corps sous forme d'une exclamation impli- 
cite, l'autre est absenté par une entrée qui mène d'emblée à la rubrique d'un dictionnaire. Du grand-père au petit-fils et de l'onomatopée au discours, c'est une sorte d'implicite réflexion sur l'origine des langues qui se réécrit là, presque sur un mode xviI $\mathrm{e}^{\mathrm{e}}$ siècle. Il y aurait d'ailleurs bien des choses à dire sur cette extraordinaire invention, en forme presque de parabole, de l'aveugle paralytique mené par son petit-fils inconnu qui voit, décrit (nomme) puis écrit (raconte) le monde de leur déambulation commune. De ces séances aussi de lecture où "Hours went by and neither one of us budged from our spots, Effing in his wheelchair, I on the sofa across from him, and there were times when I became so engrossed in what I was reading that I hardly knew where I was anymore, that I felt I was no longer sitting in my own skin." (p. 112) ${ }^{13}$

Car justement la pseudonymie intertextuelle "native" de Fogg l'inscrit tout entier sous le signe du changement de peau, de l'absorption vampirique par l'identité d'un autre, comme il advient dans toute lecture un tant soit peu intense. On revêt toujours l'identité d'un autre, comme Fogg dans le costume de Victor, on se nourrit de sa substance, et avec les livres légués par ce même Victor cela va du figuré au propre, c'est-à-dire le mouvement d'une incarnation. Mais précisément ce dernier exemple montre bien que la vampirisation va dans les deux sens: on prête aussi sa propre substance à qui vous habite. Toute lecture refait autre le livre. Toute proximité est une contagion. Cela s'appelle la solidarité, au sens étymologique qui dit l'appartenance au même volume, au même "solide", la société, le monde, la culture, la nature, et surtout l'intersection de leurs diverses sphères. Et l'on ne joue jamais qu'avec du hasard dont, comme l'oncle Victor, on fait du sens. Comme Fogg aussi, avec ces fameux livres, qu'il commence par transformer en purs volumes, cénotaphes attendris de l'oncle servant à meubler son propre espace, puis ouvrant au hasard une caisse, toutes les autres, les livres, un à un, les lisant, dans l'ordre où il les trouve et qui est celui du temps de Victor dans lequel ainsi il se glisse en lui donnant du même coup un

13 "Les heures se succédaient et nous ne bougions ni l'un ni l'autre, Effing dans son fauteuil roulant,moi sur le canapé en face de lui, et il marrivait d'être si absorbé par ce que je lisais que je ne savais plus trop où je me trouvais, que javais le sentiment de n'être plus dans ma propre peau. (Moon Palace, p. 139) 
112

"retour ", justifié de ce deuil qui est aussi une pérennité, la continuité de la vie jusque dans l'alimentaire à quoi servent en fin de compte, lus, les livres. Chaîne de sens, et chaîne nourricière, transmission, culture en un mot, dans son acception la plus noble, la plus complète et qui pourrait bien être l'acceptation de l'Autre comme impulsion et comme horizon du sujet. Il s'agit en fin de compte, comme disait en substance Mallarmé, de prendre sa place dans la chaîne des fictions qui forment cet étrange accident qu'est l'homme, et de ce hasard de l'émergence et du lieu, faire sens, c'est-à-dire mouvement, sanction et refus, répétition et déplacement.

$\mathrm{Si}$ au bout d'une course marquée par le hasard, le héros hérite d'un patronyme qui est coïncidence et le propulse dans un autre texte que sa propre vie (de fiction s'entend, c'est-à-dire que pour la lecture, l'effet intertextuel se trouve ainsi redoublé et ironisé), ce texte autre est lui-même tissé d'une pléthore d'altérités dont il devient le paradigme, du héros de Jules Verne aux explorateurs que sont Marco Polo et Stanley, dont Fogg, d'après Victor, tire ses prénoms. Autrement dit, c'est par la référence reconnue, c'est-à-dire le paradigme, la métaphore et aussi bien la mimesis, que le sens peut circuler, qu'à partir du hasard littéral une nécessité peut commander la variation et la poïesis, comme autant de formes d'une attention au monde, une attention altérante, qui apporte des "reprises", dans tous les sens du terme. Et qui reprend, surtout, ses distances.

Ressortissant plutôt au pseudonyme qu'à un jeu prénominal comme celui que nous avons vu "cratyliser" Anna et Otto, s'agissant des prénoms de Fogg, on peut aussi penser qu'à la diachronie de références au niveau de leur signifié ils combinent une référence synchrone au niveau de leur signifiant, sous la forme peut-être d'une discrète allusion à The World according to Garp, M.S. Fogg n'étant pas sans évoquer le fameux T.S. Garp du roman d'Irving. Mais à la différence du "Technical Sergeant", grade et fonction, à quoi renvoient les initiales de Garp dont le nom, on s'en souviendra n'est que l'onomatopée que mourant il laisse échapper, comme s'il n'êtait fait que des deux extrêmes: une immatriculation et un corps ${ }^{14}$, à la différence de Garp, Fogg, lui,

14 On notera la parenté avec Effing, surtout si l'on se souvient de la présenceabsence, dans son nom, des trois points, indice social, comme une immatriculation. 
n'est que variation, entre-deux, discours répercuté et ainsi ses prénoms forment système avec son nom de famille en forme de palimpseste.

Résorbés dans leurs initiales, les prénoms dont l'un, remarquons-le, provient de l'arbitraire d'un goût ("my mother had chosen Marco simply because she liked it ", p. 6-7) et l'autre de la motivation généalogique ("Stanley had been my grandfather's name", p. 7), se trouvent deux acceptions dans le commun: "Multiple Sclerosis" et "manuscript", l'une respectant les règles de l'initialisation, l'autre jouant soit le découpage étymologique soit une anarchique trouée à la Effing. Ce qui ne les empêche pas, toujours sous la forme de leurs initiales, de singer le propre d'identités illustres, "echoing the gods of modern literature" (E.M. Forster, T.S. Eliot?).

Si la famille joue la référence, forme de variation, mais aussi précision de l'amarre, si Fogg lui-même parie sur la réduction qui l'inscrit d'emblée entre son grand-père aveugle et paraplégique, diminué donc, et son père plus qu'obèse, augmenté en quelque sorte, dans une alternance que lui-même connaitra entre faim et abondance, les camarades de classe, eux, jouent la dérive et l'àpeu-près, évidemment moqueurs :

Names are the easiest thing to attack, and Fogg lent itself to a host of spontaneous mutilations: Fag and Frog, for example, along with countless meteorological references: Snowball Head, Slush Man, Drizzle Mouth. Once my last name had been exhausted, they turned their attention to the first. The $o$ at the end of Marco was obvious enough, yielding epithets such as Dumbo, Jerko, and Mumbo Jumbo, but what they did in other ways defied all expectations. Marco became Marco Polo; Marco Polo became Polo Shirt; Polo Shirt became Shirt Face; and Shirt Face became Shit face - a dazzling bit of cruelty that stunned me the first time I heard it. (p. 7) ${ }^{15}$

15 "Rien n'est plus vulnérable que les noms, et "Fogg se prêtait à une foule de mutilations spontanées: Fag et Frog, par exemple, accompagnées d'innombrables allusions météorologiques: Boule de Neige, Gadoue, Gueule de Crachin. Après avoir épuisé les ressources que leur offrait mon patronyme, mes camarades avaient dirigé leur attention sur mon prénom. Le o à la fin de Marco était assez évident pour susciter des épithètes telles que Dumbo, Jerko, et Mumbo-Jumbo, mais ce qu'ils ont trouvé en outre défiait toute attente. Marco est devenu Marco Polo; Marco Polo, Polo Shirt; Polo Shirt, Shirt Face; et Shirt Face a donné Shit Face, une éblouissante manifestation de cruauté qui m'a stupéfié la première fois que je l'ai entendue. (Moon Palace, p. 18). 
114

D'abord altérations métaplasmiques avec changement de son (quasi imperceptible dans certains accents) ou ajout de lettre qui, rappelant l'aventure de l'ancêtre oiseau parlant une autre langue, en offre du même coup la dérision dans cette grenouille qui ne fait, elle, que sauter et coasser, puis simple dérive métonymicométaphorique à partir du paradigme de fogg dont on remarquera que le parcours suit une solidification décroissante, le dernier terme étant le plus rapproché de l'initiale, comme si l'on assistait à une sorte de liquéfaction d'un bonhomme de neige, l'inscription de la bruine dans la bouche signalant à la fois les postillons et le caractère brumeux du discours, le o final du prénom, redoublant celui, central, du nom que le texte passe ici sous silence, déclenche le délire phonétique où l'assonance part de diverses nuances de l'imbécillité (dumb, jerk, avec en outre pour ce dernier terme une connotation sexuelle, masturbatoire plus précisément) pour atteindre à l'enflure (jumbo), y compris au sens injurieux que le terme peut avoir en français. Enfin l'apothéose des mots en chaîne, sur le modèle "marabout bout de ficelle", part de la référence retrouvée ("Marco Polo"), cette fois ainsi dérision de Victor, pour atteindre à la merde grâce à une altération métaplasmique jouant le lapsus avec changement de son cette fois, au contraire, plutôt marqué. Ainsi partis du rebut ("fag", mégot, peut-être aussi, abréviation de "faggot", tante, pédale), la dérive onomastique, le déchirement moral et littéral de Fogg, y reviennent, non sans avoir dans leur parcours inscrit sous une forme dérisoire non seulement les diverses lectures privées du nom mais, pourrait-on dire, toutes les ressources de la rhétorique, telles que le parler de la Halle, comme dirait Du Marsais, peut les mobiliser. Dans cette poétique carnavalesque, le personnage se voit arraché à lui-même, disséminé en une syntagmatique débridée qui cependant lui renvoie (au lecteur plutôt), ainsi qu'en un palais des glaces forain, autant d'images de lui-même, déformées certes mais suffisamment reconnaissables pour que, le voulût-il, il puisse y reconnaitre tel ou tel segment de son histoire, telle ou telle allusion à ses rêves, telle prémonition même, en forme de prolepse narrative. Ainsi texturé de part en part, lu à travers le mythe et dans la dérision, changeant de registre du familial au social, réduit ou étirê, refermé sur son mutisme ou battu de tous les vents de la variation, entre anonymat et pseudonyme, le personnage, par ce nom justement qui l'indexicalise aux yeux de la lecture et l'indexe à toutes variations qu'elle peut percevoir, assume dès lors son destin de signifiant, toujours en partance, décalé, 
pétri d'altérité et dénudé jusqu'à la trame littérale. "Le signe seul existe...provisoire", disait Jarry (César Antéchrist).

L'exil commence à la lettre, c'est aussi la leçon de Melville, notamment parce qu'à l'anonyme Ishmael se combine son "bosom friend ", le polygraphié Queequeg. Homme signe et indigène déplacé, il est à la fois l'ancêtre de The Illustrated Man de Ray Bradbury et la figure éponyme de cette peau de l'autre dans laquelle partout chez Auster un personnage se glisse. En effet, Queequeg dont le bras tatoué enserre Ishmael et se confond avec la couverture sous laquelle tous deux reposent ( $c f$. le chapitre 4 de Moby Dick, "The counterpane ") est le signe d'une adhérence chaleureuse au monde et de cette contagion en forme de solidarité dont je parlais plus haut. Queequeg enfin, qui signe en reproduisant simplement un des motifs qui ornent sa peau, pourrait bien être, tout sauvage qu'il soit, et même, d'autant plus qu'il l'est, une superbe métaphore de la culture comme pléthore de signes où chacun peut trouver sa place, fût-il un proscrit comme Ishmael, à condition paradoxalement d'accepter de la perdre et de s'ouvrir au métissage et à l's impureté . de toute syntagmatique. Queequeg ou l'anti-Léviathan, l'anti-Moby Dick.

Face au monstrueux silence de l'insigne baleine, l'hommelexique et son corps sémiotisé de part en part nous rappellent peut-être, comme Fogg et tant d'autres personnages de Paul Auster, que la dépossession d'identité librement consentie dans le babélien labyrinthe des signes est ce qui fonde l'écriture, ce partage d'un espace dont le lieu d'être s'épelle substitution. Et diaspora. 